\title{
Nanostructures and Self-Assembly of Organogels via Benzimidazole/Benzothiazole Imide Derivatives with Different Alkyl Substituent Chains
}

\author{
Xihai Shen, 1,2 Tifeng Jiao,, ${ }^{2,3}$ Qingrui Zhang, ${ }^{2}$ Haiying Guo, ${ }^{2}$ Yaopeng Lv, \\ Jingxin Zhou, ${ }^{2}$ and Faming Gao ${ }^{2}$ \\ ${ }^{1}$ College of Physics and Chemistry, Hebei Normal University of Science and Technology, Qinhuangdao 066004, China \\ ${ }^{2}$ Hebei Key Laboratory of Applied Chemistry, School of Environmental and Chemical Engineering, Yanshan University, \\ Qinhuangdao 066004, China \\ ${ }^{3}$ State Key Laboratory of Solid Lubrication, Lanzhou Institute of Chemical Physics, Chinese Academy of Sciences, \\ Lanzhou 730000, China
}

Correspondence should be addressed to Tifeng Jiao; tfiao@ysu.edu.cn

Received 15 March 2013; Accepted 28 March 2013

Academic Editor: Xingbin Yan

Copyright (C) 2013 Xihai Shen et al. This is an open access article distributed under the Creative Commons Attribution License, which permits unrestricted use, distribution, and reproduction in any medium, provided the original work is properly cited.

\begin{abstract}
New benzimidazole/benzothiazole imide derivatives with different alkyl substituent chains were designed and synthesized. Their gelation behaviors in 22 solvents were tested as novel low-molecular-mass organic gelators. The test showed that the alkyl substituent chains and headgroups of benzimidazole/benzothiazole residues in gelators played a crucial role in the gelation behavior of all compounds in various organic solvents. More alkyl chains in molecular skeletons in present gelators are favorable for the gelation of organic solvents. SEM and AFM observations revealed that the gelator molecules self-assemble into different aggregates from wrinkle, lamella and belt to dot with change of solvents. Spectral studies indicated that there existed different H-bond formation between imide groups and hydrophobic force of alkyl substituent chains in molecular skeletons. The present work may give some insights into design and character of new organogelators and soft materials with special molecular structures.
\end{abstract}

\section{Introduction}

In recent years, self-assembly and gelation properties of low-molecular-mass organic gelators have become one of the hot areas in soft matter research due to their scientific values and many potential applications in biomedical field, including tissue engineering, controlled drug release, and medical implants [1-10]. These organogels are usually considered as supramolecular gels, in which the gelator molecules self-assemble into three-dimensional networks in which the solvent is trapped via various noncovalent interactions, such as hydrogen bonding, $\pi-\pi$ stacking, van der Waals interaction, dipole-dipole interaction, coordination, solvophobic interaction, and host-guest interaction [11-15]. In order to form appropriate organogels, there are two important points. One is the design of the gelator molecules. The other is the adjustment of the external conditions.
For the former, the control of self-assembling mode at the molecular level and the balance between precipitation and dissolution in a given solvent are critical to the design of new gelators. This is because the gelation of a solvent by a gelator is the result of gelator-gelator and gelatorsolvent interactions, which involve specific and nonspecific intermolecular forces. Therefore, the aggregation behavior of a gelator in solvents can be adjusted by varying its structure.

In addition, as versatile units, the covalently bound benzimidazole/benzothiazole groups, of which one type of rings is benzene rings and the other type is a fivemember ring with $\mathrm{N}$ or $\mathrm{S}$ elements, have been widely chosen for designing new amphiphiles because of their unique directional self-association through $\pi-\pi$ stacking and van der Waals interactions in the process of supramolecular 
assembly [16-18]. For example, some benzimidazole and benzothiazole derivatives formed chiral assembly films through a cooperative stereoregular $\pi-\pi$ stacking of the functional groups together with the long alkyl chains in a helical sense [18]. Therein the relationship between the chirality of assembly films and the molecular structures of amphiphiles as well as their H-bond or coordination behaviors was discussed. In our reported work, the gelation properties of some cholesterol imide derivatives consisting of cholesteryl units and photoresponsive azobenzene substituent groups have been investigated [19]. We found that a subtle change in the headgroup of azobenzene segment can produce a dramatic change in the gelation behavior of both compounds. In addition, the gelation properties of bolaform and trigonal cholesteryl derivatives with different aromatic spacers have been characterized [20]. Therein, we have investigated the spacer effect on the microstructures of such organogels and found that various kinds of hydrogen bond interactions among the molecules play an important role in the formation of gels.

In this paper, as a continuous work, we have designed and synthesized new benzimidazole/benzothiazole imide derivatives with different alkyl substituent chains. We have found that all compounds could form different organogels in various organic solvents. Characterization of the organogels by SEM and AFM revealed different structures of the aggregates in the gels. We have investigated the effect of alkyl substituent chains and headgroups residues in gelators on the microstructures of such organogels in detail and found different kinds of hydrogen bond interactions between intermolecular imide groups.

\section{Experimental}

2.1. Materials. The starting materials, 2 -aminobenzimidazole and 2-aminobenzothiazole, were purchased from Alfa Aesar Tianjin Chemicals. Other used reagents were all for analysis purity from TCI Shanghai Chemicals or Aldrich Chemicals, respectively. The solvents were obtained from Beijing Chemicals and were distilled before use. Deionized water was used in all cases. 4-Hexadecyloxybenzoic acid and 3,4,5-tris(hexadecyloxy)benzoic acid were synthesized in our laboratory according to previous report [21] and confirmed by ${ }^{1} \mathrm{H}$ NMR. Then, these imide objectives were prepared by simple methods. Simply speaking, different benzoic acid chlorides were synthesized by heating acid compounds solution in sulfoxide chloride and a bit of DMF for about $10 \mathrm{~h}$ at $70^{\circ} \mathrm{C}$. Then, the prepared benzoic acid chlorides reacted with corresponding amines in dried dichloromethane at the presence of pyridine for 2 days at room temperature. After that, the mixtures were washed with dilute hydrochloric acid and pure water. The organic layer was evaporated to dryness. The residues were purified by recrystallization in ethanol solution as pale or yellow solids. The final products and their abbreviations are shown in Figure 1, which were confirmed by ${ }^{1} \mathrm{H}$ NMR and elemental analysis.

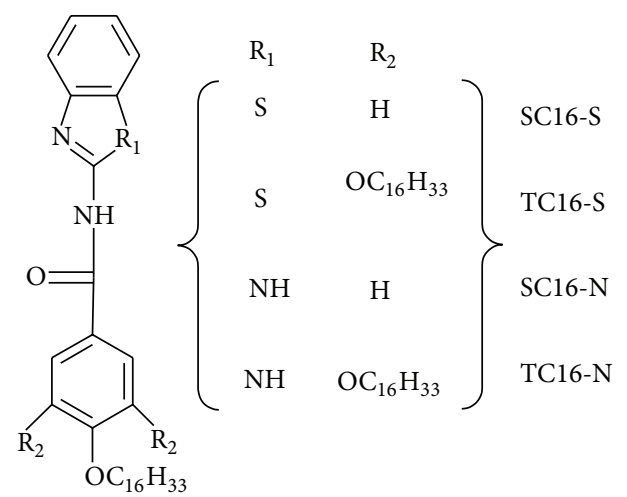

FIGURE 1: Structures and abbreviations of these imide derivatives with different substituent groups.

2.2. Gelation Test. A weighted amount of gelator and a measured volume of selected pure organic solvent were placed into a sealed glass bottle and the solution was heated in a water bath until the solid was dissolved. Then, the solution was cooled to room temperature in air, and the test bottle was inversed to see if a gel was formed. When the gelator formed a gel by immobilizing the solvent at this stage, it was denoted as "G." For the systems in which only solution remained until the end of the tests, they were referred to as solution (S). The system in which the potential gelator could not be dissolved even at the boiling point of the solvent was designated as an insoluble system (I). Critical gelation concentration (CGC) refers to the minimum concentration of the gelator for gel formation.

2.3. Measurements. Firstly, the xerogel was prepared by a vacuum pump for $12-24 \mathrm{~h}$. The dried sample thus obtained was attached to mica, copper foil, glass, and $\mathrm{CaF}_{2}$ slice for morphological and spectral investigation, respectively. Before SEM measurement, the samples were coated on copper foil fixed by conductive adhesive tape and shielded by gold. SEM pictures of the xerogel were taken on a Hitachi S-4800 field emission scanning electron microscopy with the accelerating voltage of $5-15 \mathrm{kV}$. AFM images were recorded using Nanoscope VIII Multimode Scanning Probe Microscope (Veeco Instrument, USA) with silicon cantilever probes. All AFM images were shown in the height mode without any image processing except flattening. Transmission FT-IR spectra of the xerogel were obtained by Nicolet is/10 FT-IR spectrophotometer from Thermo Fisher Scientific Inc. by average 32 scans and at a resolution of $4 \mathrm{~cm}^{-1}$. The XRD measurement was conducted using a Rigaku D/max 2550PC diffractometer (Rigaku Inc., Tokyo, Japan). The XRD pattern was obtained using $\mathrm{CuK} \alpha$ radiation with an incident wavelength of $0.1542 \mathrm{~nm}$ under a voltage of $40 \mathrm{kV}$ and a current of $200 \mathrm{~mA}$. The scan rate was $0.5^{\circ} / \mathrm{min} .{ }^{1} \mathrm{H}$ NMR spectra was obtained on a Bruker ARX-400 (Bruker, Inc., Switzerland) NMR spectrometer in $\mathrm{CDCl}_{3}$ with TMS as an internal standard. The elemental analysis was carried out with the Flash EA Carlo-Erba-1106 Thermo-Quest. 


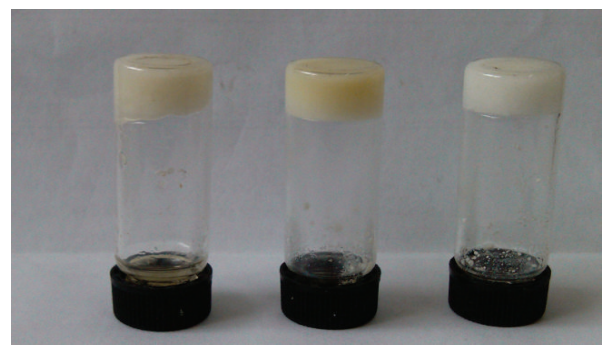

(a)

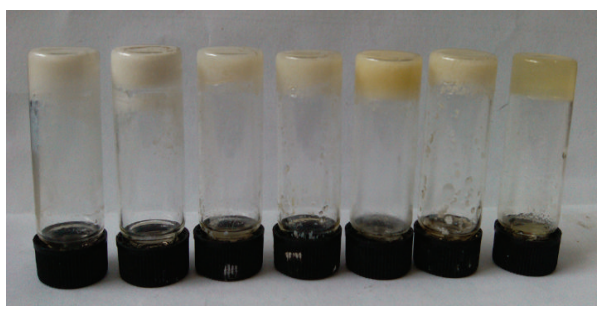

(c)

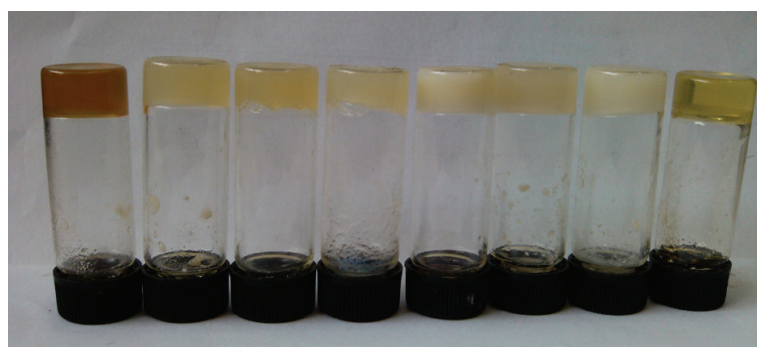

(b)

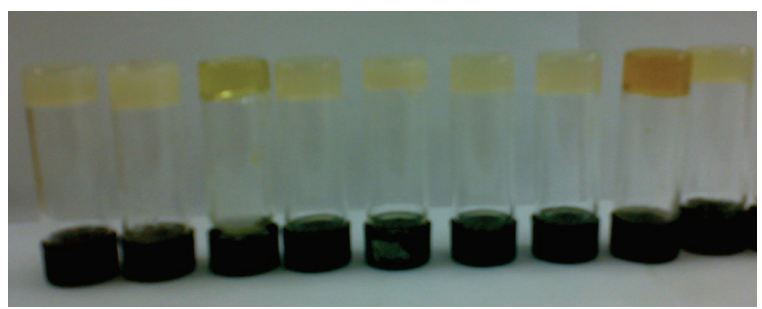

(d)

FIGURE 2: Photographs of organogels in different solvents: (a) SC16-S; (b) TC16-S; (c) SC16-N; (d) TC16-N.

TABLE 1: Gelation behaviors of all compounds at room temperature. ${ }^{a}$

\begin{tabular}{lcccc}
\hline Solvents & SC16-S & TC16-S & SC16-N & TC16-N \\
\hline Acetonitrile & I & I & I & I \\
1,4-Dioxane & S & I & I & G \\
Isopentanol & I & G & I & G \\
n-Butyl acrylate & I & I & G & S \\
Benzene & S & S & S & S \\
Formaldehyde & I & I & I & I \\
n-Butanol & G & G & I & G \\
Petroleum ether & I & S & G & I \\
Nitrobenzene & S & G & G & G \\
Tetrachloromethane & S & S & S & S \\
Acetone & S & I & G & I \\
Cyclohexanone & S & I & G & S \\
Cyclopentanone & I & I & G & I \\
n-Propanol & G & G & I & G \\
Pyridine & S & S & I & S \\
THF & S & S & S & S \\
DMF & I & G & I & G \\
Ethyl acetate & I & G & I & G \\
Ethanolamine & G & I & G & I \\
Isopropanol & I & G & I & G \\
n-Hexane & I & S & I & S \\
Aniline & I & G & I & G \\
\hline
\end{tabular}

${ }^{a}$ S: solution; G: gel; I: insoluble.

\section{Results and Discussion}

3.1. Gelation Behaviors of These Imide Derivatives. The gelation performances of all compounds in 22 solvents are listed in Table 1. Examination of the table reveals that all compounds are efficient gelators. Firstly, SC16-S with benzothiazole segment and single alkyl substituent chain in molecular skeleton can gel in 3 solvents, including n-butanol, $\mathrm{n}$-propanol, and ethanolamine, respectively. As for TC16-S with three alkyl substituent chains in molecular skeletons, 8 kinds of organogels were formed, such as isopentanol, n-butanol, nitrobenzene, n-propanol, DMF, ethyl acetate, isopropanol, and aniline, respectively. Secondly, as for SC16$\mathrm{N}$ and TC16-N with benzimidazole segment in molecular skeleton, the numbers of formed organogels changed to 7 and 9, respectively. The photographs of all organogels in different solvents were shown in Figure 2. The data shown in Table 1 indicate that the change of functional headgroups and alkyl substituent groups can have a profound effect upon the gelation abilities of these studied compounds. It seemed that more alkyl chains in molecular skeletons in present gelators are more favorable for the present benzothiazole imide derivatives in gelation of organic solvents than those of benzimidazole compounds. In addition, the headgroup effect of benzimidazole/benzothiazole for intermolecular stacking in the gel formation process is also obvious for all cases. It obviously indicated that SC16-N, benzimidazole derivative with single alkyl substituent chain in molecular skeleton, can form more gels than SC16-S. The reasons for the strengthen on the gelation behaviors can be assigned to the change of intermolecular hydrogen bonding and spatial conformation of the gelators due to benzimidazole/benzothiazole and more alkyl substituent chains in molecular skeletons [22, 23] which may increase the ability of the gelator molecules to selfassemble into ordered structures, a necessity for forming organized assembly structures.

3.2. Morphological Investigation of Organogels. In addition, in order to obtain a visual insight into the gel microstructures, 


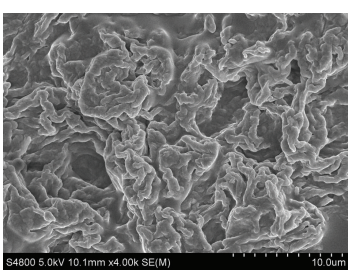

(a)

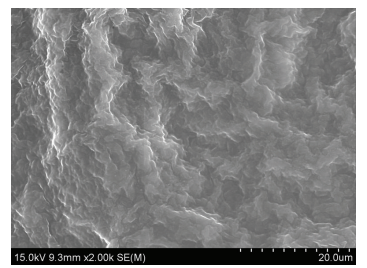

(e)

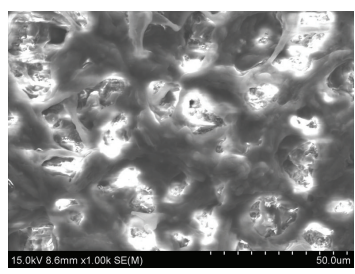

(b)

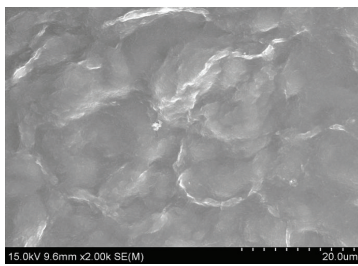

(f)

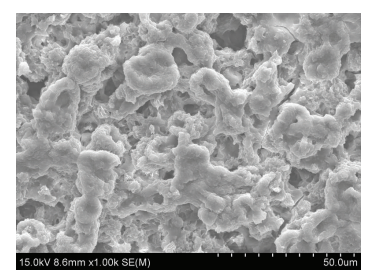

(c)

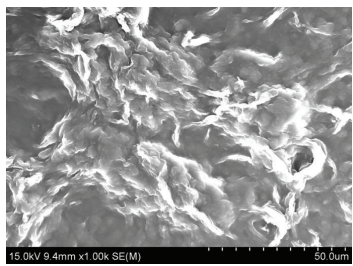

$(\mathrm{g})$

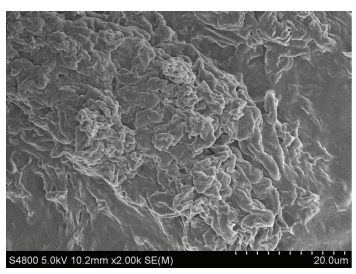

(d)

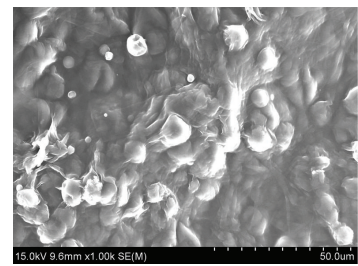

(h)

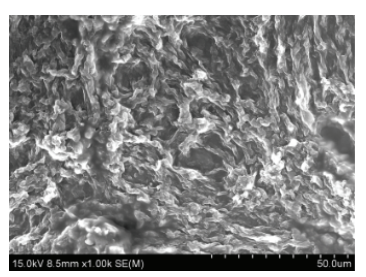

(i)

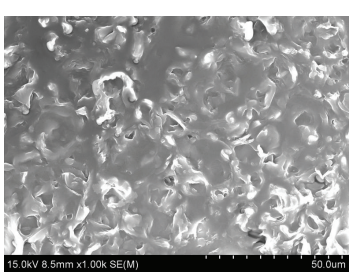

(j)

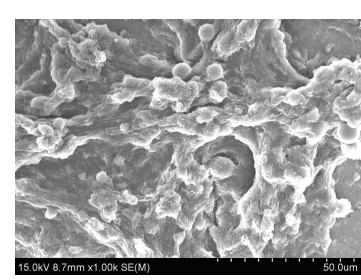

(k)

FIGURE 3: SEM images of xerogels from SC16-S gels ((a) n-butanol; (b) n-propanol; (c) ethanolamine, resp.) and TC16-S gels ((d) isopentanol; (e) n-butanol; (f) nitrobenzene; (g) n-propanol; (h) DMF; (i) ethyl acetate; (j) isopropanol; (k) aniline, resp.).

the typical nanostructures of the xerogels were studied by SEM technique, as shown in Figures 3 and 4 . From the present diverse images, it can be easily investigated that the microstructures of the xerogels of all compounds in different solvents are significantly different from each other, and the morphologies of the aggregates change from wrinkle, lamella and belt to dot with change of solvents. In addition, more regular belt-like or fiber-like aggregates with different aspect ratios were prepared in gels of TC16-S and TC16-N with three alkyl substituent chains in molecular skeletons. As for two other compounds with single alkyl substituent chains, most of the aggregates tended to be wrinkled or deformed films. Furthermore, the xerogels of SC16-S and TC16-S in n-butanol, with SC16-N and TC16$\mathrm{N}$ in nitrobenzene, were characterized by $\mathrm{AFM}$, as shown in Figure 5. From the images, it is interesting to note that these big belt or lamella aggregates were composed of many little rod-like or needle-like nanodomains by stacking of the present imide derivatives. The morphologies of the aggregates shown in the SEM and AFM images may be rationalized by considering a commonly accepted idea that highly directional intermolecular interactions, such as hydrogen bonding or $\pi-\pi$ interactions, favor formation of belt or fiber micro/nanostructures [24-26]. The difference of morphologies between molecules with single/multialkyl substituent chains can be mainly due to the different strengths of the intermolecular hydrophobic force between alkyl substituent chains, which have played an important role in regulating the intermolecular orderly staking and formation of special aggregates.

3.3. Spectral Investigation of Organogels. In addition, in order to further investigate the orderly stacking of xerogels nanostructures, XRD of all compounds xerogels from gels were measured. Firstly, the data of TC16-S was taken as an example, as shown in Figure 6(a). The curve for TC16-S xerogel from DMF shows main peaks in the angle region $(2 \theta$ values, $4.85^{\circ}, 6.45^{\circ}, 9.72^{\circ}, 11.36^{\circ}, 19.65^{\circ}, 21.66^{\circ}$, and $23.56^{\circ}$ ) corresponding to $d$ values of $1.82 \mathrm{~nm}, 1.37 \mathrm{~nm}, 0.91 \mathrm{~nm}$, $0.78 \mathrm{~nm}, 0.45 \mathrm{~nm}, 0.41 \mathrm{~nm}$, and $0.38 \mathrm{~nm}$, respectively. The corresponding $d$ values of $1.82 \mathrm{~nm}, 0.91 \mathrm{~nm}, 0.45 \mathrm{~nm}$, and $0.38 \mathrm{~nm}$ follow a ratio of $1: 1 / 2: 1 / 4: 1 / 5$, suggesting a lamellarlike structure of the aggregates in the gel [27]. In addition, the XRD data of xerogels of SC16-S and TC16-S in n-butanol, with SC16-N and TC16-N in nitrobenzene, were compared, as shown in Figure 6(b). As for the curve of SC16-S in n-butanol, strong peaks appeared in the angle region ( $2 \theta$ values, $4.10^{\circ}$, $6.12^{\circ}, 8.22^{\circ}, 12.30^{\circ}, 16.35^{\circ}, 18.90^{\circ}$, and $20.46^{\circ}$ ) corresponding to $d$ values of $2.16 \mathrm{~nm}, 1.45 \mathrm{~nm}, 1.08 \mathrm{~nm}, 0.72 \mathrm{~nm}, 0.54 \mathrm{~nm}$, $0.47 \mathrm{~nm}$, and $0.43 \mathrm{~nm}$, respectively. The corresponding $d$ values of $2.16 \mathrm{~nm}, 1.08 \mathrm{~nm}, 0.72 \mathrm{~nm}, 0.54 \mathrm{~nm}$, and $0.43 \mathrm{~nm}$ follow a ratio of $1: 1 / 2: 1 / 3: 1 / 4: 1 / 5$, suggesting a good lamellar-like structure of the aggregates in the gel. For three other curves, little relative weak peaks were obtained, suggesting polycrystalline or amorphous nanostructures. The XRD results described above demonstrated again that the alkyl substituent 


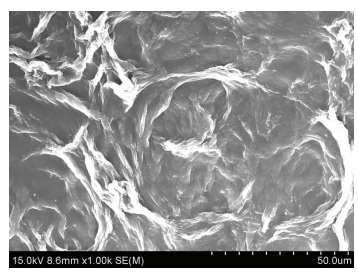

(a)

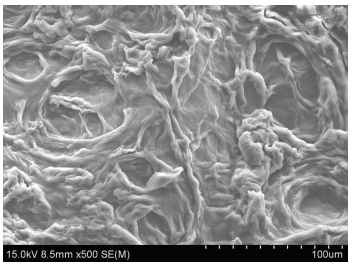

(e)

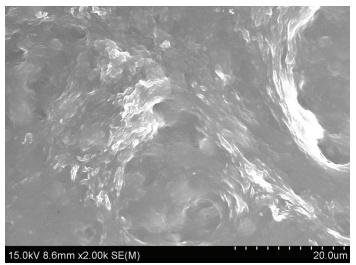

(i)

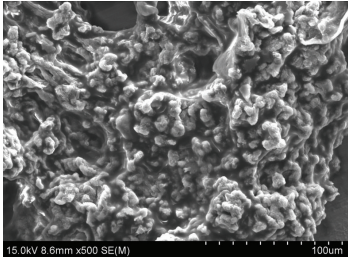

(m)

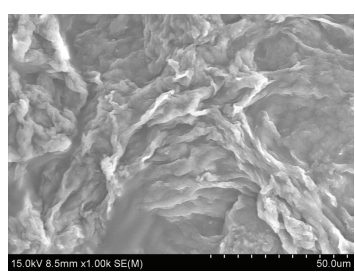

(b)

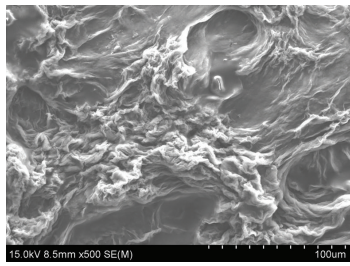

(f)

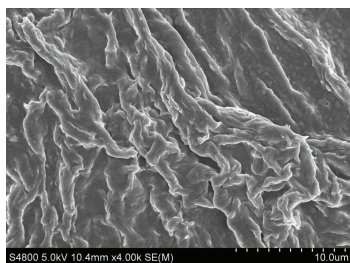

(j)

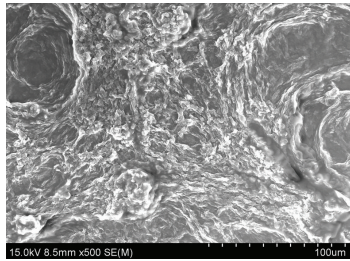

(n)

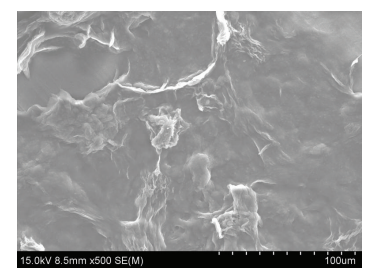

(c)

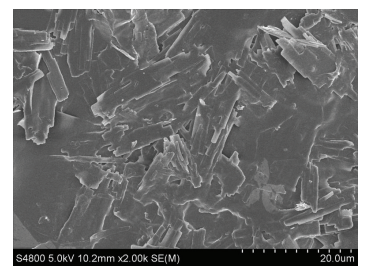

(g)

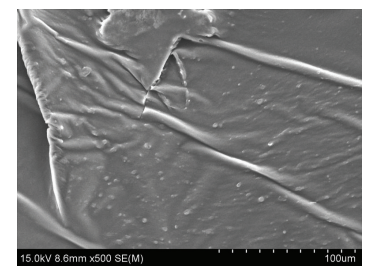

(k)

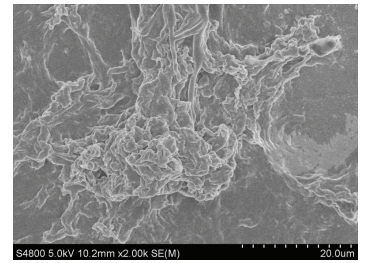

(o)

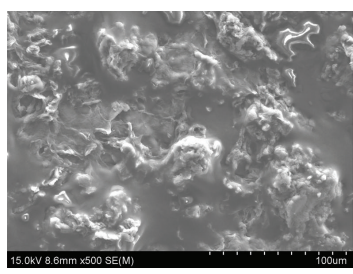

(d)

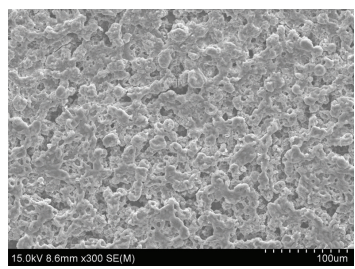

(h)

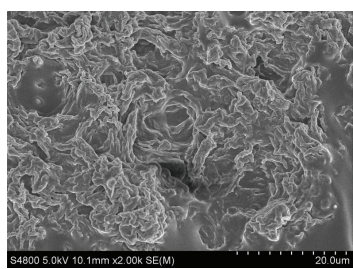

(1)

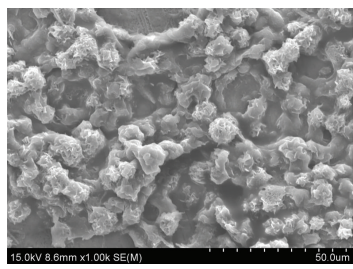

(p)

FIGURE 4: SEM images of xerogels from SC16-N gels ((a) n-butyl acrylate; (b) petroleum ether; (c) nitrobenzene; (d) acetone; (e) cyclohexanone; (f) cyclopentanone; (g) ethanolamine, resp.) and TC16-N gels ((h) 1,4-dioxane; (i) isopentanol; (j) n-butanol; (k) nitrobenzene; (l) n-propanol; (m) DMF; (n) ethyl acetate; (o) Isopropanol; (p) aniline, resp.).

chains had a great effect on the assembly modes of these imide compounds.

It is well-known that hydrogen bonding plays an important role in the formation of organogels $[28,29]$. At present, in order to further clarify this and investigate the effect of substituent groups on assembly, we have measured the FT-IR spectra of xerogels of all compounds. Firstly, TC16$\mathrm{S}$ was taken as an example, as shown in Figure 7(a). As far as spectra of these xerogels, some main peaks were observed at $3242,2918,2848,1678$, and $1468 \mathrm{~cm}^{-1}$, respectively, which can be assigned to the N-H stretching, methylene stretching, amide I band, and methylene scissoring, respectively [30, 31]. These bands indicate $\mathrm{H}$-bond formation between intermolecular imide groups in the gel state. In addition, the spectra of xerogels of all compounds in n-butanol and nitrobenzene were compared, as shown in Figure 7(b). One obvious change is the decrement of methylene stretching for SC16$\mathrm{S}$ and SC16-N in comparison with other two compounds, which can be attributed to the number difference of alkyl substituent chains in molecular skeletons. Another change is that the peaks assigned to N-H stretching for SC16-N and TC16-N shifted to 3305 and $3212 \mathrm{~cm}^{-1}$, respectively. This implied that there were differences in the strength of the intermolecular hydrogen-bond interactions in these xerogels, even though they were from the same solvent system [32-34].

\section{Conclusions}

In summary, some benzimidazole/benzothiazole imide derivatives with different alkyl substituent chains have been synthesized. Their gelation behaviors in various organic solvents can be regulated by changing alkyl substituent chains and headgroups of benzimidazole/benzothiazole segment. The experimental data demonstrated that the numbers of alkyl substituent chains linked to benzene ring in these imide derivatives can have a profound effect upon the gelation abilities of these studied compounds. More alkyl chains in 


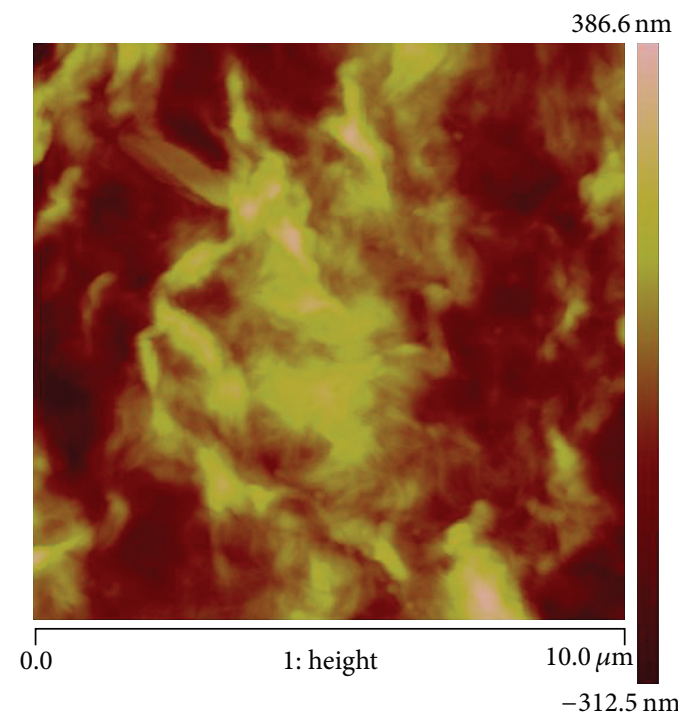

(a)

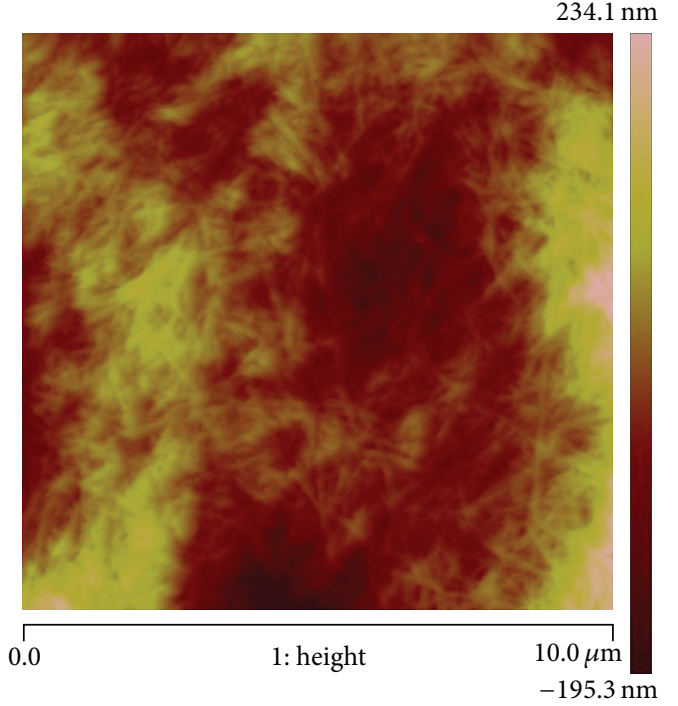

(c)

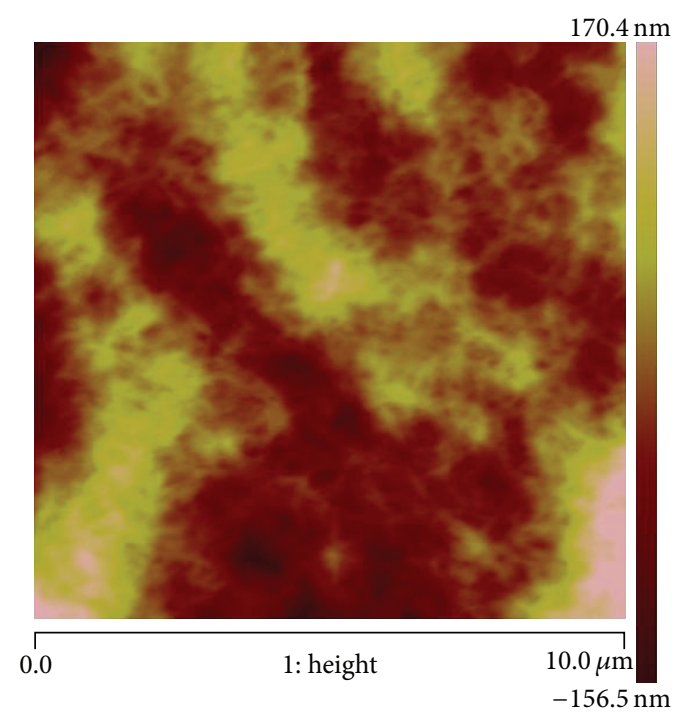

(b)

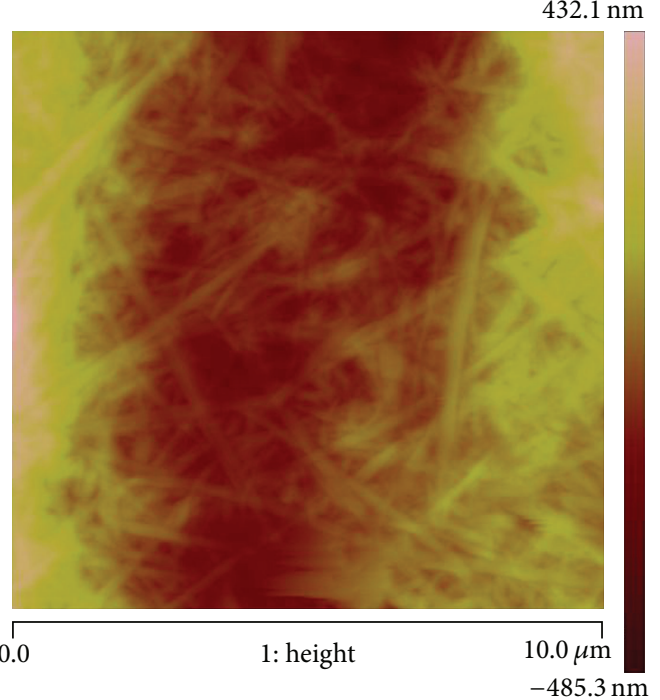

(d)

FIGURE 5: AFM images of xerogels: SC16-S (a) and TC16-S (b) gels in n-butanol, respectively; SC16-N (c) and TC16-N (d) gels in nitrobenzene, respectively.

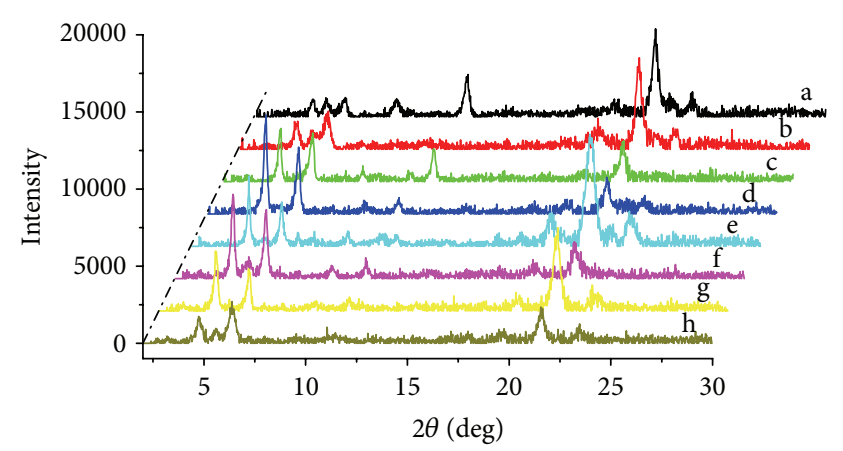

(a)

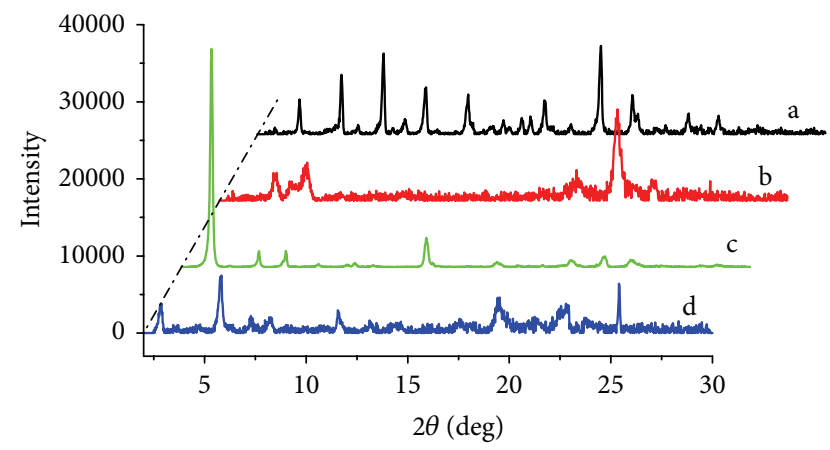

(b)

FIGURE 6: X-ray diffraction patterns of xerogels: (a) TC16-S (a: isopentanol; b: n-butanol; c: nitrobenzene; d: n-propanol; e: DMF; f: ethyl acetate; g: isopropanol; h: aniline, resp.); (b) SC16-S (a) and TC16-S (b) gels in n-butanol, respectively; SC16-N (c) and TC16-N (d) gels in nitrobenzene, respectively. 


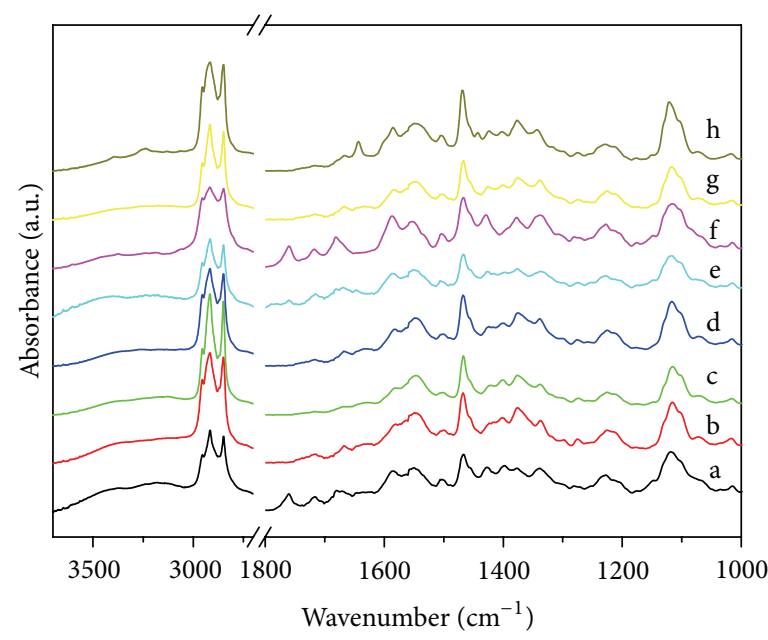

(a)

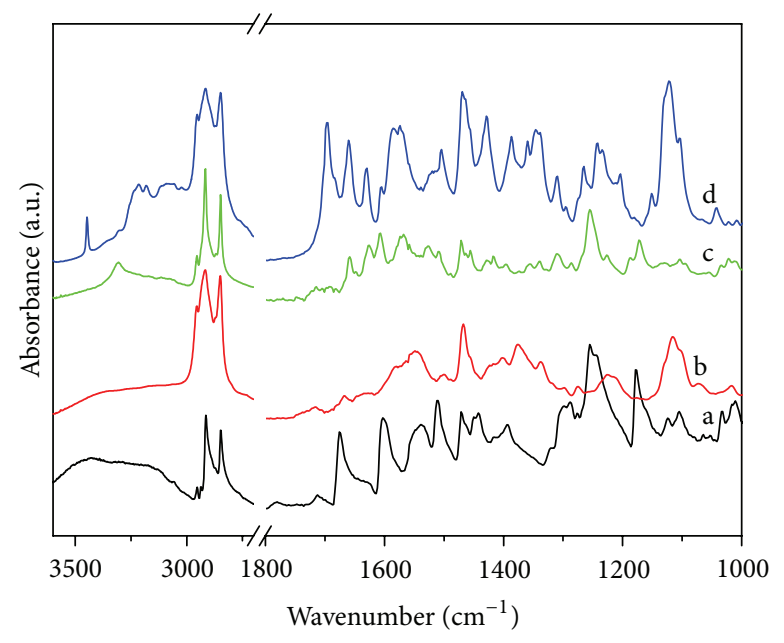

(b)

FIGURE 7: FT-IR spectra of xerogels: (a) TC16-S (a: isopentanol; b: n-butanol; c: nitrobenzene; d: n-propanol; e: DMF; f: ethyl acetate; g: isopropanol; h: aniline, resp.); (b) SC16-S (a) and TC16-S (b) gels in n-butanol, respectively; SC16-N (c) and TC16-N (d) gels in nitrobenzene, respectively.

molecular skeletons in present gelators are favorable for the gelation of organic solvents. Morphological studies revealed that the gelator molecules self-assemble into different aggregates from wrinkle, lamella and belt to dot with change of solvents. Spectral studies indicated that there existed different $\mathrm{H}$-bond formation and hydrophobic force, depending on benzimidazole/benzothiazole segment and alkyl substituent chains in molecular skeletons. The present research work affords new useful exploration for the design and development of new versatile low-molecular-mass organogelators and soft matter.

\section{Conflict of Interests}

The authors declare that they have no any direct financial relation with the commercial identities mentioned in this paper that might lead to a conflict of interest for any of the authors.

\section{Acknowledgments}

This work was financially supported by the National Natural Science Foundation of China (Grant nos. 20903078 and 21207112), the Natural Science Foundation of Hebei Province (Grant nos. B2012203060 and B2013203108), the China Postdoctoral Science Foundation (Grant nos. 2011M500540 and 2012M510770), the Support Program for Hundred Excellent Innovation Talents from Universities and Colleges of Hebei Province (Grant no. CPRC020), the Science Foundation for the Excellent Youth Scholars from Universities and Colleges of Hebei Province (Grant no. Y2011113), the Scientific Research Foundation for Returned Overseas Chinese Scholars of Hebei Province (Grant no. 2011052), and the Open Foundation of State Key Laboratory of Solid Lubrication (Lanzhou Institute of Chemical Physics, CAS) (Grant no. 1002).

\section{References}

[1] P. Xing, T. Sun, S. Li, A. Hao, J. Su, and Y. Hou, "An instant-formative heat-set organogel induced by small organic molecules at a high temperature," Colloids and Surfaces A, vol. 421, pp. 44-50, 2013.

[2] F. Xin, H. Zhang, B. Hao et al., "Controllable transformation from sensitive and reversible heat-set organogel to stable gel induced by sodium acetate," Colloids and Surfaces A, vol. 410, pp. 18-22, 2012.

[3] K. Iwanaga, T. Sumizawa, M. Miyazaki, and M. Kakemi, "Characterization of organogel as a novel oral controlled release formulation for lipophilic compounds," International Journal of Pharmaceutics, vol. 388, no. 1-2, pp. 123-128, 2010.

[4] M. Löfman, J. Koivukorpi, V. Noponen, H. Salo, and E. Sievänen, "Bile acid alkylamide derivatives as low molecular weight organogelators: systematic gelation studies and qualitative structural analysis of the systems," Journal of Colloid and Interface Science, vol. 360, no. 2, pp. 633-644, 2011.

[5] G. Bastiat, F. Plourde, A. Motulsky et al., "Tyrosine-based rivastigmine-loaded organogels in the treatment of Alzheimer's disease," Biomaterials, vol. 31, no. 23, pp. 6031-6038, 2010.

[6] Z.-G. Tao, X. Zhao, X.-K. Jiang, and Z.-T. Li, "A hexaazatriphenylene-based organogel that responds to silver(I) with high selectivity under aqueous condition," Tetrahedron Letters, vol. 53, no. 14, pp. 1840-1842, 2012.

[7] X. Yu, Y. Li, Y. Yin, and D. Yu, "A simple and colorimetric fluoride receptor and its fluoride-responsive organogel," Materials Science and Engineering C, vol. 32, no. 6, pp. 1695-1698, 2012.

[8] M. Takizawa, A. Kimoto, and J. Abe, "Photochromic organogel based on [2.2]paracyclophane-bridged imidazole dimer with tetrapodal urea moieties," Dyes and Pigments, vol. 89, no. 3, pp. 254-259, 2011.

[9] M. Xue, D. Gao, X. Chen, K. Liu, and Y. Fang, "New dimeric cholesteryl-based A(LS)2 gelators with remarkable gelling abilities: organogel formation at room temperature," Journal of Colloid and Interface Science, vol. 361, no. 2, pp. 556-564, 2011. 
[10] F. Delbecq, K. Tsujimoto, Y. Ogue, H. Endo, and T. Kawai, "N-stearoyl amino acid derivatives: potent biomimetic hydro/ organogelators as templates for preparation of gold nanoparticles," Journal of Colloid and Interface Science, vol. 390, no. 1, pp. 17-24, 2013.

[11] X. Ren, W. Yu, Z. Zhang et al., "Gelation and fluorescent organogels of a complex of perylenetetracarboxylic tetraacid with cationic surfactants," Colloids and Surfaces A, vol. 375, no. 1-3, pp. 156-162, 2011.

[12] P. He, J. Liu, K. Liu et al., "Preparation of novel organometallic derivatives of cholesterol and their gel-formation properties," Colloids and Surfaces A, vol. 362, no. 1-3, pp. 127-134, 2010.

[13] W. Zhao, Y. Li, T. Sun et al., "Heat-set supramolecular organogels composed of $\beta$-cyclodextrin and substituted aniline in N,N-dimethylformamide," Colloids and Surfaces A, vol. 374, no. 1-3, pp. 115-120, 2011.

[14] H. Svobodová, Nonappa, Z. Wimmer, and E. Kolehmainen, "Design, synthesis and stimuli responsive gelation of novel stigmasterol-amino acid conjugates," Journal of Colloid and Interface Science, vol. 361, no. 2, pp. 587-593, 2011.

[15] Y. Huang, J. Ge, Z. Cai, Z. Hu, and X. Hong, "The correlation of microstructure morphology with gelation mechanism for sodium soaps in organic solvents," Colloids and Surfaces A, vol. 414, pp. 88-97, 2012.

[16] M. Liu, A. Kira, and H. Nakahara, "Silver(I) ion induced monolayer formation of 2-substituted benzimidazoles at the air/water interface," Langmuir, vol. 13, no. 18, pp. 4807-4809, 1997.

[17] M. Liu and J. Cai, "Silver(I) ion induced reverse U-shape monolayers of poly(methylenebis(benzimidazoles)) at the air/water interface," Langmuir, vol. 16, no. 6, pp. 2899-2901, 2000.

[18] P. Guo and M. Liu, "Fabrication of chiral Langmuir-Schaefer films of achiral amphiphilic Schiff base derivatives through an interfacial organization," Langmuir, vol. 21, no. 8, pp. 3410-3412, 2005.

[19] T. Jiao, Y. Wang, F. Gao, J. Zhou, and F. Gao, "Photoresponsive organogel and organized nanostructures of cholesterol imide derivatives with azobenzene substituent groups," Progress in Natural Science, vol. 22, no. 1, pp. 64-70, 2012.

[20] T. Jiao, F. Gao, Y. Wang, J. Zhou, F. Gao, and X. Luo, "Supramolecular gel and nanostructures of bolaform and trigonal cholesteryl derivatives with different aromatic spacers," Current Nanoscience, vol. 8, no. 1, pp. 111-116, 2012.

[21] H. Yang, T. Yi, Z. Zhou et al., "Switchable fluorescent organogels and mesomorphic superstructure based on naphthalene derivatives," Langmuir, vol. 23, no. 15, pp. 8224-8230, 2007.

[22] F. R. Lupi, D. Gabriele, V. Greco, N. Baldino, L. Seta, and B. de Cindio, "A rheological characterisation of an olive oil/fatty alcohols organogel," Food Research International, vol. 51, no. 2, pp. 510-517, 2013.

[23] C. Zhao, B. Bai, H. Wang et al., "Self-assemblies, helical ribbons and gelation tuned by solvent-gelator interaction in a bi-1,3,4oxadiazole gelator," Journal of Molecular Structure, vol. 1037, pp. 130-135, 2013.

[24] M. K. Nayak, "Functional organogel based on a hydroxyl naphthanilide derivative and aggregation induced enhanced fluorescence emission," Journal of Photochemistry and Photobiology A, vol. 217, no. 1, pp. 40-48, 2011.

[25] T. Atsbeha, L. Bussotti, S. Cicchi et al., "Photophysical characterization of low-molecular weight organogels for energy transfer and light harvesting," Journal of Molecular Structure, vol. 993, no. 1-3, pp. 459-463, 2011.
[26] G. Zhu and J. S. Dordick, "Solvent effect on organogel formation by low molecular weight molecules," Chemistry of Materials, vol. 18, no. 25, pp. 5988-5995, 2006.

[27] J. Wu, T. Yi, Q. Xia et al., "Tunable gel formation by both sonication and thermal processing in a cholesterol-based selfassembly system," Chemistry, vol. 15, no. 25, pp. 6234-6243, 2009.

[28] T. Shimizu and M. Masuda, "Stereochemical effect of evenodd connecting links on supramolecular assemblies made of 1glucosamide bolaamphiphiles," Journal of the American Chemical Society, vol. 119, no. 12, pp. 2812-2818, 1997.

[29] M. Kogiso, S. Ohnishi, K. Yase, M. Masuda, and T. Shimizu, "Dicarboxylic oligopeptide bolaamphiphiles: proton-triggered self-assembly of microtubes with loose solid surfaces," Langmuir, vol. 14, no. 18, pp. 4978-4986, 1998.

[30] T. Wang, Y. Li, and M. Liu, "Gelation and self-assembly of glutamate bolaamphiphiles with hybrid linkers: effect of the aromatic ring and alkyl spacers," Soft Matter, vol. 5, no. 5, pp. 1066-1073, 2009.

[31] Y. Li, T. Wang, and M. Liu, "Ultrasound induced formation of organogel from a glutamic dendron," Tetrahedron, vol. 63, no. 31, pp. 7468-7473, 2007.

[32] J. L. Gurav, I. K. Jung, H. H. Park, E. S. Kang, and D. Y. Nadargi, "Silica aerogel: synthesis and applications," Journal of Nanomaterials, vol. 2010, Article ID 409310, 11 pages, 2010.

[33] M. Suárez, A. Fernández, J. L. Menéndez, and R. Torrecillas, "Transparent yttrium aluminium garnet obtained by spark plasma sintering of lyophilized gels," Journal of Nanomaterials, vol. 2009, Article ID 138490, 5 pages, 2009.

[34] A. S. Al Dwayyan, M. Naziruddin Khan, and M. S. Al Salhi, "Optical characterization of chemically etched nanoporous silicon embedded in sol-gel matrix," Journal of Nanomaterials, vol. 2012, Article ID 713203, 7 pages, 2012. 

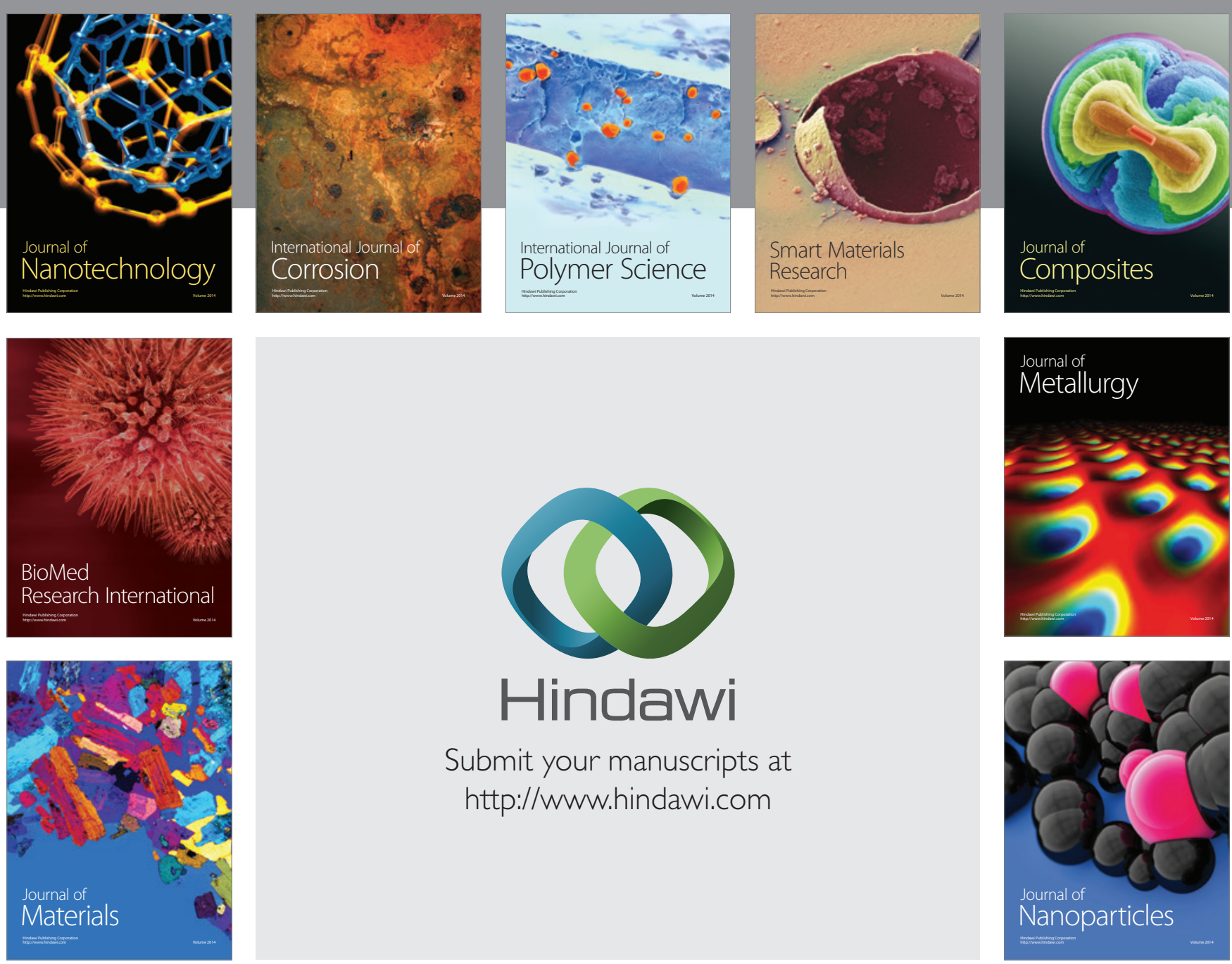

Submit your manuscripts at http://www.hindawi.com
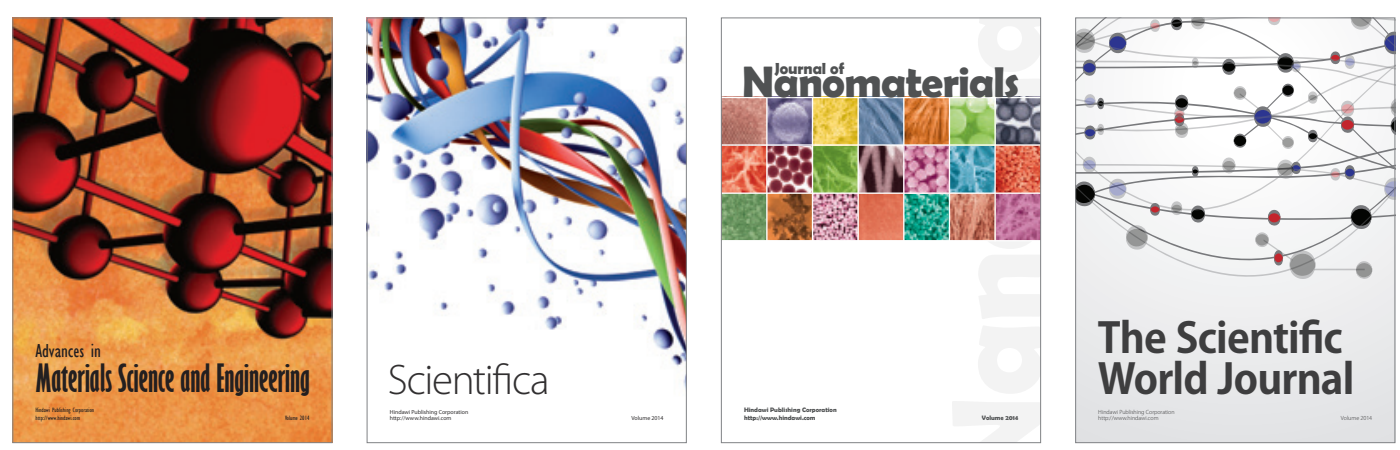

\section{The Scientific World Journal}
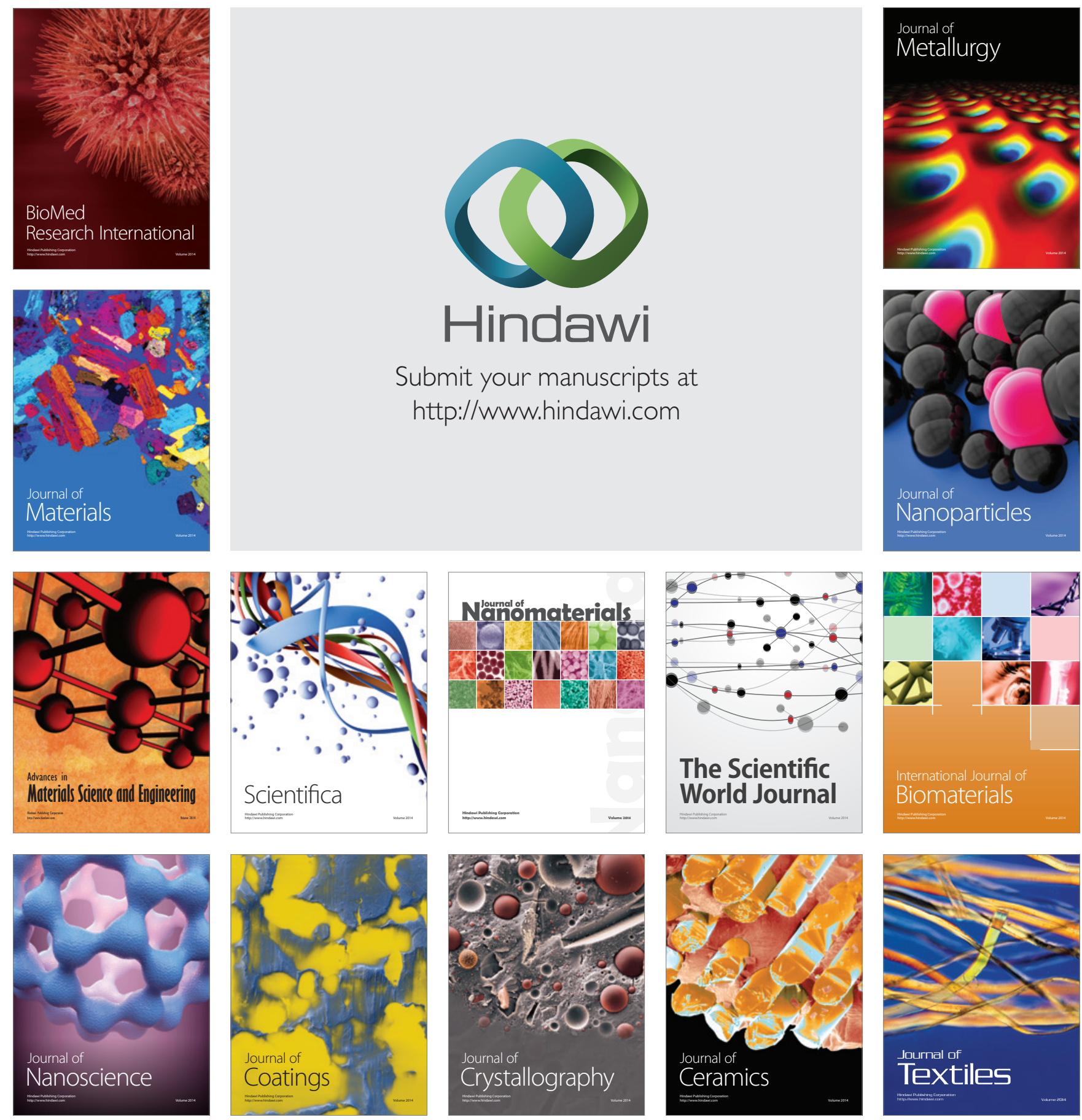